\title{
Research on BP Neural Network Motor Control Algorithm Based on Frog Leaping Algorithm
}

\author{
Liu Xia, Zhang Lili and Wang Hanlin \\ School of Mechanical and Materials Engineering, Xi'an University,710065
}

Keywords: PID Control; Motor Control; BP Neural Network; Frog Leaping Algorithm

\begin{abstract}
Traditional PID motor control methods have problems of low control accuracy and slow response speed. Therefore, in order to improve the performance of the speed control of brushless DC motor, a control method based on BP neural network and shuffled frog leaping algorithm is proposed. The DC motor was first modeled. Then, a 4-layer structure BP neural network is used to realize multilayer perception. Finally, the complete steps of motor control are realized by using shuffled frog leaping algorithm. The simulation results show that the proposed motor control method has faster response speed, higher control accuracy and better reliability and effectiveness compared with the traditional PID control method.
\end{abstract}

\section{Introduction}

The position of the electric motor in modern social economic development has been continuously improved [1]. The demand for high-performance motor control systems is also increasing. Due to its long service life, low maintenance cost, small size and noise, DC motors are widely used in various modern advanced electronic devices, such as printers, cameras, washing machines, CNC machine tools, rudders, drones and robots [2]. The traditional PID control method is mainly used to solve the linear time-invariant problem, in which the parameters need to be set in advance and cannot be changed with the change of the control object. However, the brushless DC motor usually has a large nonlinearity, so the brushless DC motor control system based on the traditional PID control method has the problems of poor anti-interference ability and low precision of speed control [3]. In order to improve the performance of brushless DC motor control, researchers proposed many intelligent control methods, such as neural network control and fuzzy control [4]. In reference [5], a speed-loop fuzzy PID controller is proposed, which can overcome the shortcomings of traditional PID controllers and realize double closed-loop control of brushless DC motors. In reference [6], a control method of brushless DC motor based on improved particle swarm optimization is proposed, which can achieve higher response speed and stability. In reference [7], a neural network-based control method for brushless DC motors is proposed, which can adapt to environmental changes and realize any nonlinear function approximation by learning. Therefore, in order to further improve the performance of BLDCM speed control, a BLDCM control method based on BP neural network and shuffled frog leaping algorithm is proposed based on the above research theory. The simulation results show that the proposed motor control method has faster response speed, higher control accuracy and better reliability and effectiveness compared with the traditional PID control method.

\section{System Model of Brushless DC Motor}

This hypothesis assumes that the three-phase armature windings of the motor stator in brushless DC motors have the same parameters, differ by $120^{\circ}$ and are completely symmetrical [8]. The voltage equation of the brushless DC motor is showing as below. 


$$
\left[\begin{array}{l}
u_{a} \\
u_{b} \\
u_{c}
\end{array}\right]=\left[\begin{array}{lll}
R_{s} & 0 & 0 \\
0 & R_{s} & 0 \\
0 & 0 & R_{s}
\end{array}\right]\left[\begin{array}{l}
i_{a} \\
i_{b} \\
i_{c}
\end{array}\right]+\left[\begin{array}{lll}
L_{s} & L_{m} & L_{m} \\
L_{m} & L_{s} & L_{m} \\
L_{m} & L_{m} & L_{s}
\end{array}\right] p\left[\begin{array}{c}
i_{a} \\
i_{b} \\
i_{c}
\end{array}\right]+\left[\begin{array}{l}
e_{a} \\
e_{b} \\
e_{c}
\end{array}\right]
$$

$u_{a}, u_{b}$ and ${ }^{u_{c}}$ respectively represents the voltages of the three stator windings, in units of V. ${ }^{i_{a}},{ }^{i_{b}}$ and $i_{c}$ represents the current of a single stator. ${ }^{R_{s}}$ represents the resistance of a single stator, in units of $\Omega .{ }^{L_{s}}$ represents the inductance of a single stator, in units of $\mathrm{H} .{ }^{L_{m}}$ represents the mutual inductance between the stator windings, in units of $\mathrm{H}$. P represents the differential operator. $e_{a} 、 e_{b}$ and $e_{c}$ respectively represents the back electromotive force of the three stator windings. According to the above digital-analogue model, this paper uses Simulink to implement the brushless DC motor system simulation model in Matlab7.0 environment, as shown in formula 1. The system simulation model uses a double closed-loop speed control mechanism to simulate load and speed changes. The key step in system simulation is to obtain a back-EMF trapezoidal wave.

\section{The Frog Leaping Algorithm BP Neural Network Control Design}

The Structure of BP Neural Network. This paper adopts BP neural network to realize multilayer perception. The proposed BP neural network consists of a four-layer structure, which includes input layer, variable layer, fuzzy rule layer and output layer, as shown in Figure 1.

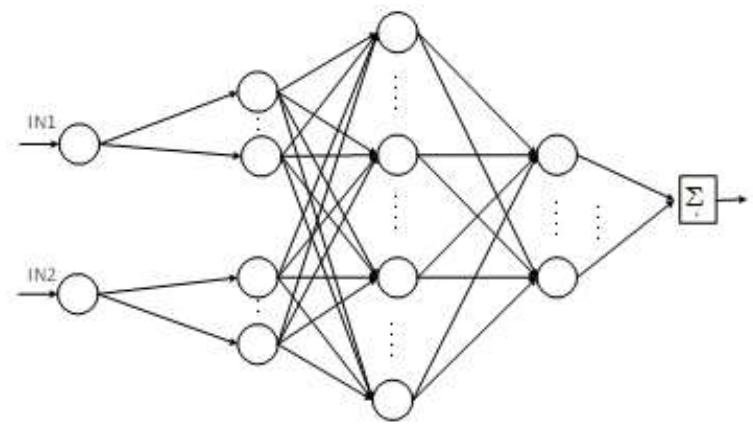

Figure 1 BP neural network structure diagram

In the first layer (input layer), the input of the IN1 input node is the error after the transformation, and the input of the IN2 input node is the rate of change of the error after the transformation. In the variable layer, the signal of the input layer is classified by using a Gaussian function. Rule level is used for input and output transformations. The output layer is used for defuzzification.

Shuffled Frog Leaping Algorithm. In a local space, the Shuffled frog-leaping algorithm (SFLA) achieves jump feeding by simulating the biological evolutionary behavior of frog populations. Shuffled frog-leaping algorithm is a novel sub heuristic swarm intelligence simulation algorithm, which has the advantages of fast convergence, simple principle, easy implementation, less adjustable parameters and global optimization. Therefore, many researchers have been constantly trying to optimize the SFLA algorithm in order to enhance its performance in various practical applications. Most of these optimization methods improve the performance of prediction or control by modifying the frog group initialization parameters and the weight of the search step, updating the iteration order or combining other algorithms. In reference [9], it combines shuffled frog leaping algorithm and neural network to avoid local defects and achieve higher accuracy of mechanical motor fault diagnosis. At the present stage, various hybrid optimization schemes composed of shuffled frog leaping algorithms perform well in solving various optimization problems.

The key step of shuffled frog-leaping algorithm is the evolution process of locally search. This step uses iterative calculations. The updated logic of the frog population during each iteration is as follows. 


$$
\begin{aligned}
& D_{i}=\operatorname{rand}() *\left(x^{b}-x^{w}\right), i=1,2, \ldots, m \\
& x^{w}=x^{w}+D_{i},-D_{\text {max }} \leq D_{i} \leq D_{\text {max }}, i=1,2, \ldots, m
\end{aligned}
$$

$\operatorname{rand}()$ denotes a random coefficient with a range of $(0,1) . D_{i}$ shows the distance that the individual jumps each time. $D_{\max }$ represents the maximum distance the individual can jump. $x^{b}$ and $x^{w}$ denotes respectively the frog individuals with the best and worst match values in the secondary population. The worst-fitting frog uses equation (3) to update the individual's location data. If the updated result is better than $x^{w}$, the updated result is stored and treated as a new result. If the updated result is not better than $x^{w}$, then continue to perform equations (2) and (3) to update the worst result.

Motor Control Steps Based on SFLA-BP Neural Network. This article uses the SFLA-BP neural network to implement the brushless DC motor control steps showing as follows.

Step 1: Select the initial motor control data sample to determine the parameters of SFLA and BP neural network;

Step 2: For all training sample sets, a better solution is generated by the BP neural network and copied $\mathrm{m}$ times, and then an F-m solution is generated randomly;

Step 3: Calculate the fit value of each frog and sort it in descending order;

Step 4: Perform a local search according to the adaptive mobile search strategy, and then update the worst solution in each subpopulation;

Step 5: Determine whether the updated solution has become better. If yes, skip to step 8; otherwise, perform subsequent steps.

Step 6: Use the population optimal to replace the sub-population optimal solution, update the local search to update the worst solution;

Step 7: Determine whether the updated solution is still better. If yes, skip to step 8. Otherwise, perform the following steps.

Step 8: Randomly generate a solution and replace the original worst solution with the new solution.

Step 9: Determine if the number of local iterations reaches the maximum value. If yes, perform subsequent steps; otherwise, skip to step 4.

Step 10: The secondary population is mixed and ready to enter the next iteration, and then it is judged whether the termination condition is satisfied. If yes, then the optimal result is output. Otherwise, skip to step 3 to continue the calculation of the fitting value of each frog.

\section{Results and Analysis of Simulation Experiments}

In order to analyze and verify the SFLA-BP neural network motor control algorithm presented in this paper, specific motor simulation experiments are carried out. The motor simulation parameters are showing as follows: rated voltage of $250 \mathrm{~V}$, rated speed of $1000 \mathrm{rad} / \mathrm{min}$, back electromotive force coefficient of $0.394 \mathrm{~V} /(\mathrm{rad} . \mathrm{s}-1)$, phase inductance of $0.005 \mathrm{H}$, resistance of 1 and moment of inertia of $0.005 \mathrm{~kg}$. M2. The motor rotation load is $1 \mathrm{Nm}$ and the given rotation speed is $1000 \mathrm{r} / \mathrm{min}$. 

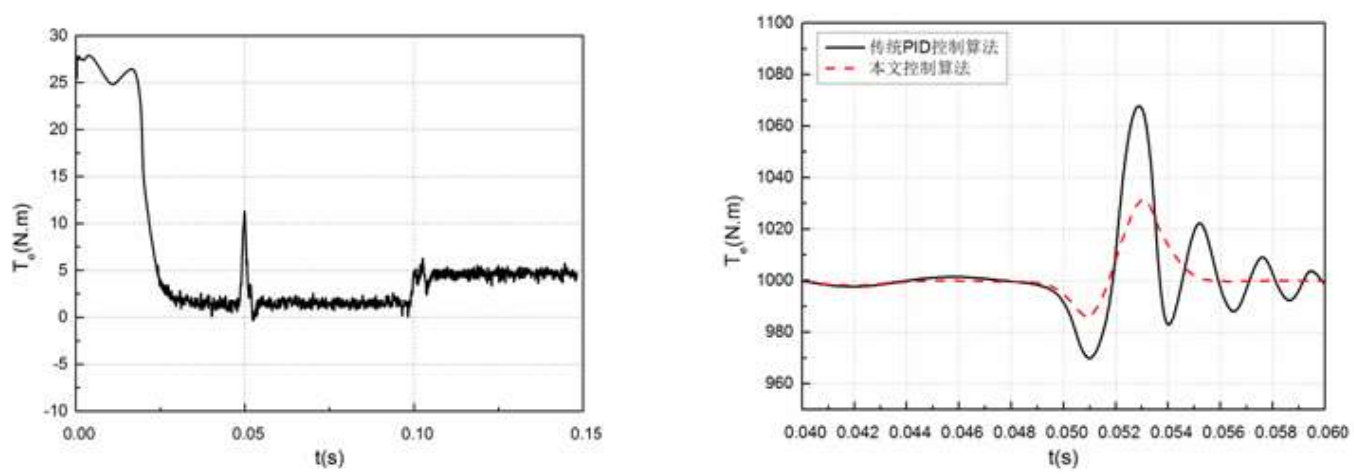

Fig. 2 The torque response output of the control algorithm Fig. 3 Comparison result of the speed response output

The torque response output of the control algorithm in this paper is shown in figure 2 . In order to compare and analyze the proposed algorithm, under the condition of experimental parameters, this paper compares the algorithm with the traditional PID control algorithm [8]. The result of the comparison between the two different algorithms for the speed response output is shown in fig. 2 . As it can be seen from figure 3, this algorithm's speed response output has no overshoot and has a higher accuracy and faster response speed compared with the traditional traditional PID algorithm.

\section{Conclusion}

A BLDCM control method based on BP neural network and shuffled frog leaping algorithm is proposed. The DC motor was first modeled. Then, a 4-layer structure BP neural network is used to realize multilayer perception. Finally, the complete steps of motor control are realized by using shuffled frog leaping algorithm. The simulation results show that the proposed motor control method has faster response speed, higher control accuracy and better reliability and effectiveness compared with the traditional PID control method.

\section{Problems and Improvements in Teaching}

The human society in the 21 st century has experienced rapid development of informationization, and the speed of social rhythms and knowledge growth has greatly increased. In the context of this rapid development of information technology, new demands are placed on our higher education. At present, talents required by the market should have the following five skills and qualities: basic knowledge learning skills, information acquisition skills, innovative skills, interpersonal skills and practical skills. Basic knowledge learning skills refer to your ability to acquire specific knowledge. Information acquisition skills refer to the ability to obtain relevant information through specific means and possess certain analytical information. Innovative skills means that you can develop on the basis of the original learning. The ability of interpersonal communication mainly refers to the quality of teamwork in division of labor. Practical ability mainly refers to having strong practical and hands-on capabilities. At present, our country proposes to take a new road to industrialization and drive the development level of the entire industry through the improvement of the level of informatization. On this basis, higher education has been given a new historical mission.

The automation major is a engineering major with wide-ranging, widely-oriented and multi-demanding. It is an applied science produced in practice. This major not only requires students to have a strong theoretical foundation, but also requires students to have strong practical ability and innovative ability. Therefore, practical teaching is a very important part of the automation profession. Over the years, our country's automation profession has been continuously developing and has made remarkable achievements in education and teaching. But overall, there is still a big gap between us and our foreign counterparts. Our curriculum system is not standardized. Compared with teaching methods and teaching requirements, we are still lagging behind. In the level of professional understanding, we still have the drawbacks of emphasizing theoretical and 
light practice. The main manifestation is that practical teaching is attached to theoretical teaching. Practical teaching is often separated from the engineering environment, and it is out of touch with the actual situation. In the experimental methods, most of the experiments focus on the theory of verification, and there is not enough emphasis on the students' experimental ideas and experimental design. The experimental equipment of many colleges and universities is also relatively backward. The quality of the graduation design is generally not high, and many schools even regard the graduation design of students as the flow. Therefore, the quality of graduation design is not fully guaranteed. Besides, students' production internships are often not fully guaranteed. Although some schools have practice bases, it is difficult for students to actually get training opportunities in practice bases, and it is difficult to truly participate in the design and production of enterprise products. The existence of these problems has seriously hampered the improvement of students' comprehensive literacy and their ability to innovate.

We should give full attention to the above problems and actively adopt effective countermeasures. First of all, we should establish a systematic and modular curriculum system and constantly enrich our teaching methods. The theoretical course should be based on a knowledge system, in which the proportion of modern theoretical knowledge should be increased. The technical courses should be based on mature and widely-used technologies, eliminating the obsolete content and increasing the follow-up of modern automation technology. We should set up some special courses according to the actual conditions of the school and encourage teachers to reform the teaching content. The new achievements and new methods for the development of science and technology are introduced into the classroom teaching and curriculum experiment as much as possible. In the professional post curriculum, teachers will focus on the integrated reform of learning, use, and innovation. Encourage teachers to choose flexible and diverse teaching methods, actively use the advantages of modern information technology and communicate with students through Internet and other means to help students solve their practical difficulties in learning. Second, we should standardize the management of teaching and put the stability and improvement of teaching quality at the top of education. A dynamic, multi-level and multi-channel teaching quality monitoring system was constructed to evaluate and monitor course teaching. Third, a set of excellent, time-based, scientific and diversified teaching materials was formulated by professional and technical leaders and experienced teachers based on the teaching plans and syllabus of the discipline. Finally, we must vigorously promote the reform of practical teaching and make education and teaching meet the requirements of the training of applied talents. In the construction of the practical teaching system, we must focus on the cultivation of application ability and engineering quality, proceed from reality and constantly explore the relationship among knowledge, ability and quality. The construction of the practical teaching content system adopts the construction mode of "layered cultivation, progressive progress and gradual improvement" to create "three platforms", namely, Engineering and technology basic training platform, professional skills and ability training platform, comprehensive application ability and innovation ability training platform. On the basis of scientific analysis and determination of students' knowledge, skills and abilities and quality structure, teachers should carefully select and arrange practical courses and teaching links. In education and teaching, teachers should also pay attention to "five combinations." Namely, the combination of professional and technical education and engineering quality education, foundation of professional disciplines and the cultivation of practical ability, in-class teaching and extracurricular education, school professional education and national certification of certified engineers and teaching, scientific research and production and application-specific features.

\section{Acknowledgement}

Fund Project: National Training Programs of Innovation and Entrepreneurship for Undergraduates Project Number: 201611080015 


\section{Reference}

[1] L. F. Kong, R. Jing, F. D. Zhao and others. Research on Dynamic Clustering of Multi-mobile Robot WSN Data Collection Method[J]. Miniature Microcomputer Systems, 2014, 35(4): 845-849.

[2] S. L. Li. A Study on the Traditional Control of Motor Synchronization Based on PID Algorithm[J]. Automation and Instrumentation, 2016(11):17-18.

[3] Wang X and Tao F. Position Sensorless Control of BLDC Motor Based on Global Fast Terminal Sliding Mode Observer[J]. Transactions of China Electrotechnical Society, 2017, 15(6):1559-1566.

[4] L. Wang, G. G. Chen and Y. S. Zhao. The Design of Brushless DC Motor Controller Based on BP Neural Network[J]. Large Electric Machine Technology, 2012(2):19-21.

[5] J. W. Li, S. Cui, L. Q. Li and others. Design and Development of Brushless DC Motor Control System Based on Fuzzy PID[J]. Machine Design and Manufacture, 2013(2):77-79.

[6] H. F. Chen, H. Song and Z. Q. Wang. An Improved Brushless DC Motor Control Method[J]. Electric Machines and Control, 2015(1):28-35.

[7] S. F. Chen and X. H. Chen. Application of Neural Network in Brushless DC Motor Control System[J]. Electric Machines and Control, 2014(4):12-16.

[8] Thomas N and Poongodi P. Position Control of DC Motor Using Genetic Algorithm Based PID Controller[J]. Lecture Notes in Engineering \& Computer Science, 2009, 2177(1):121-124.

[9] Q. Zhang, S. H. Xu and P. C. Li. Fault Diagnosis of Pumping Unit Based on Quantum Frog Leaping Algorithm and Process Neural Network[J]. China Mechanical Engineering, 2014, 25(12):1609-1615. 